\title{
Kunst und Kreativität in Haft - Folgerungen aus einer Umfrage
}

Kai Bammann / Johannes Feest

\begin{abstract}
Kunst im Strafvollzug - geht das zusammen? Auf den ersten Blick scheint dies widersprüchlich zu sein, schnell stellt man jedoch fest, dass es vielfältige Möglichkeiten gibt, sich kreativ zu betätigen und im Grunde nichts dagegen spricht, dies auch im Strafvollzug zu tun.
\end{abstract}

\section{m Jahr 1981 veröffentlichten Neu- enhausen und Mitarbeiter eine im Auftrag des Bundesministeriums der Justiz erstellte Studie über die im Strafvollzug der Bundesrepublik Deutschland vorhandenen Kunst- Bundesländer gestartet mit dem Ziel Auskunft darüber zu bekommen, ob und wenn ja welche Angebote künstlerischer Betätigung es in den Haftanstalten der jeweiligen Länder gibt.} gruppen und künstlerischen Angebote $^{1}$. Neuenhausen, selbst Maler und Bildhauer, hatte einige Jahre zuvor (dazu weiter unten) selbst ein Kunstprojekt mit Strafgefangenen gestartet. Die Verfasser fanden in den damals 124 bundesdeutschen Haftanstalten insgesamt 80, in denen es künstlerische Aktivitäten gab. Eingehender dargestellt sind in der Studie 20 zu jener Zeit laufende oder geplante Projekte aus allen Bereichen der Kunst, namentlich „Malen, Zeichnen, Modellieren, Drucken und Werken“, "Theater und Laienspiel“ sowie „Film und Fernsehen“. Neuenhausen u.a. gingen mithin von einem weiten Begriff der Kunst aus und suchten vor allem auch nach Projekten, die von Menschen betreut wurden, die sich selbst als Künstler verstanden. Dabei leitete die Verfasser auch ihre eingangs der Studie gestellte Frage, ob Kunst im Strafvollzug „Luxus oder Notwendigkeit“ sei. Einige der Projekte gibt es in der dargestellten Form heute noch, andere sind verschwunden bzw. längst durch vergleichbare neue Projekte ersetzt worden. Zwischenzeitlich hat sich mit der Wiedervereinigung, einer teilweise deutlich verschärften Kriminalpolitik und einem anderen Umgang mit Strafgefangenen vieles verändert $^{2}$, so dass ein aktueller Überblick zu der Frage fehlte, wie es heute im Strafvollzug mit der Kunst bestellt ist.

\section{Forschungsidee und Fragestellung}

Mitte 2006 wurde am Strafvollzugsarchiv $^{3}$ an der Universität Bremen eine kleine Umfrage bei den Landesministerien der Justiz der 16

Gefragt wurde erstens danach, welche Gelegenheiten es für Gefangene gibt, sich in ihrer Freizeit kreativ bzw. künstlerisch auszudrücken. Insbesondere interessierte dabei auch, ob es im Rahmen der Freizeit Kunstgruppen in den Anstalten gibt, die entweder von MitarbeiterInnen des Vollzuges oder von externen Gruppen angeboten werden und wie diese Gruppen organisiert sind.

Zweitens wurde nach den Möglichkeiten für die Gefangenen gefragt, im Rahmen der Arbeitspflicht des $§ 41$ StVollzG in künstlerischen Werkbetrieben entlohnt tätig zu werden oder sich nach $§ 39$ Abs. 2 StVollzG selbstbeschäftigt künstlerischen Projekten zu widmen.

Eine dritte Frage zielte darauf $\mathrm{ab}$ in Erfahrung zu bringen, ob es kunst-therapeutische Angebote in den Haftanstalten gibt.

\section{Ergebnisse aus der Befragung der Landesjustizministerien}

Rückmeldungen kamen aus allen Bundesländern. Es stellte sich jedoch schnell heraus, dass es bezüglich „Kunstgruppen“ oder kreativer Betätigung keine Vorgaben der Lan- desministerien gab, sondern die entsprechenden Angebote in der Verantwortung und unter der Aufsicht der jeweiligen Anstalt stattfinden. Aus mehreren Antwortschreiben ist jedoch deutlich hervorgegangen, dass die Ministerien dem kreativen Tätigsein in Haft nicht nur sehr aufgeschlossen gegenüber stehen, sondern dies auch für unbedingt förderungswürdig erachten. Wie sich weiter zeigte, gibt es in allen Ländern vielfältige Angebote, die sich weder leicht vergleichen, noch unter einen gemeinsamen Nenner bringen lassen. Tatsächlich lassen sich die Projekte auch nicht auf einzelne Bereiche der Kunst eingrenzen, sondern sie reichen von der bildenden Kunst (Malen, Zeichnen, Bildhauerei, Druckgrafik, Plastizieren) über die darstellende Kunst (Theater, Tanz und HipHop) bis hin zur Musik (Chor, Gitarren- und anderer Musik-Unterricht). In der JVA Bonn gab es Ende der 1980er Jahre z.B. auch eine Eurythmiegruppe ${ }^{4}$, was als Hinweis auf die Breite der Möglichkeiten verstanden werden kann. Ergänzt werden diese Angebote durch Multimediaprojekte, Foto- und Videogruppen sowie, klassischer, durch Schreibwerkstätten.

Einer der Referenten wies in seinem Antwortschreiben ausdrücklich auf die besondere Bedeutung kreativen Tätigseins in der Haft hin. Er betonte nachdrücklich dass es bei entsprechenden Angeboten zum einen um die Stärkung der Persönlichkeit gehe und der Freizeitbereich (in den diesen Aktivitäten fallen) als Trainingsfeld für Kommunikation, Interaktion und Kooperation diene sowie ein Lernfeld für individuelles und soziales Verhalten sowie Hilfsmittel des sozialen Trainings sei. Wichtig sei auch, mit ständigen wie kurzzeitigen Angeboten dafür Sorge $\mathrm{zu}$ tragen, dass die Gefangenen neue Fertigkeiten lernten und bereits vorhandene verbesserten. Aus einem anderen Bundesland wurde sehr auf die dort angebotenen $\mathrm{Mu}-$ sikgruppen hingewiesen, die auch dazu dienten „vor der Haft erworbene künstlerische Fähigkeiten zu erhalten".

\section{Künstlerisches Arbeiten und Arbeitspflicht}

Wie zu den einzelnen Angeboten, konnten die Ministerien auch zu der Frage nach einer Einbindung künstlerischer Tätigkeit in die Arbeitspflicht keine detaillierte Auskunft geben. Generell ist die Frage, wo und wie ein Gefangener beschäftigt wird, den Vollzugsanstalten überlassen. Ausdrücklich geschaffene künstlerische Angebote, mit denen die Gefangenen ihrer Arbeitspflicht nachkommen können, gibt es allerdings auch seitens der Anstalten überwiegend nicht. Eine Ausnahme bildet hier Bremen, wo es mit der Bildhauerwerkstatt ein Kunstprojekt gibt, in dem die Gefangenen angestellt und mit Entlohnung tätig sind. Hamburg hat auf zwei Kreativ-Gruppen hingewiesen, bei denen die Teilnahme vergütet wird: eine Maltherapie in der Sozialtherapie Hahnöfersand und eine Gruppe „Kreatives Gestalten“ im Frauenstrafvollzug in Glasmoor. In Thüringen gilt entsprechendes für einen Förderlehrgang im Jugendvollzug, bei dem mit Holz, Ton, Textil und Korbwaren gearbeitet wird. Die Arbeit einer Bildhauerwerkstatt in der JVA Bruchsal (Baden-Württemberg) wurde eingestellt. Dies gilt auch für ein befristet mit EU-Mitteln gefördertes Projekt im Jugendstrafvollzug des Landes Brandenburg.

Grundsätzlich anders zu beurteilen sind Betriebe, die kunsthandwerkliche Gegenstände herstellen, 
welche dann auch zum Verkauf vorgesehen sind. Anders als Kunstwerkstätten oder an der Rehabilitation ausgerichtete Kreativgruppen sind diese wirtschaftlich ausgerichtet. Hier sind die Gefangenen wie in anderen Werkbetrieben auch als Arbeiter mit der üblichen Entlohnung und in Erfüllung der gesetzlichen Arbeitsverpflichtung tätig. Angebote dieser Art finden sich u.a. in Bayern, Niedersachsen, dem Saarland, Sachsen und Thüringen. Auch in anderen Bundesländern werden mittlerweile Produkte aus Werkstätten der JVAen verkauft, dies dann vermutlich aber eher als Handwerk verstanden und nicht im künstlerischen Bereich angesiedelt.

\section{Künstlerisches Arbeiten im Rahmen der Selbstbeschäftigung}

Die ergänzende Frage nach der Möglichkeit, ob Gefangene als Künstler selbstbeschäftigt (im Sinne des § 39 Abs. 2 StVollzG) tätig sein dürfen, konnte nicht beantwortet werden, da es sich hierbei um Einzelfallentscheidungen der JVAen handelt und die Landesjustizministerien hier in der Regel nicht unmittelbar beteiligt sind. Aus Korrespondenz mit dem Strafvollzugsarchiv, bzw. aus älterer Rechtsprechung sind jedoch einige solcher Fälle in deutschen Haftanstalten bekannt.

\section{Wenige Planstellen für KunsttherapeutInnen}

Im Übrigen haben die Landesjustizministerien, sofern sie sich in ihren Rückmeldungen hierzu geäußert haben, überwiegend die Frage verneint, ob es ausdrücklich zum Zweck der Kunsttherapie angestellte KunsttherapeutInnen in den Justizvollzugsanstalten des jeweiligen Bundeslandes gibt ${ }^{5}$. Ausführliche Informationen kamen aus Sachsen, wo in drei Haftanstalten (Zeithain zwei Stellen, Torgau und Waldheim je eine Stelle) insgesamt vier KunsttherapeutInnen beschäftigt sind. In Brandenburg ist ein Kunsttherapeut in der Erwachsenensozialtherapie tätig, allerdings nicht auf einer entsprechend ausgewiesenen Stelle. Nordrhein-Westfalen unterhält eine Kunsttherapie im Frauenstraf- vollzug in der JVA Köln, nähere Informationen gab es hier allerdings nicht.

\section{Informationen aus Gefangenenzeitungen}

Ausführlichere Informationen erhält man, wenn man die Anstalten direkt befragt, bzw. auch wenn man Blicke in die Gefangenenzeitungen wirft. Hier finden sich immer wieder Erfahrungsberichte von Gefangenen, die an Kunstprojekten teilgenommen haben.

Aus den Gefangenenzeitungen ist, anders als bei der an die Ministerien adressierten Umfrage, auch $\mathrm{zu}$ erfahren, dass es deutlich mehr KunsttherapeutInnen im Vollzug gibt. Allerdings bleiben hier die Angaben zur Organisation bzw. zur Finanzierung ungenau; da die Ministerien insoweit Planstellen verneint haben, ist jedoch von einer externen Finanzierung auszugehen. In Bremen besteht z.B. ein kunsttherapeutisches Angebot im Erwachsenenvollzug, das über einen freien Träger der Straffälligenhilfe läuft und in einem größeren mit EU-Mitteln finanzierten befristeten Projekt angesiedelt ist. In der JVA BerlinHakenfelde gibt es ein ebenfalls mit Drittmitteln gefördertes, kunsttherapeutisch ausgerichtetes Projekt, bei dem sich die Teilnehmer alle zwei Wochen für zwei Stunden zum gemeinsamen Arbeiten, aber auch Lernen treffen ${ }^{6}$. Oft finden sich auch Menschen, die ehrenamtlich entsprechende Projekte anbieten und durchführen bzw. die selbst für ihre Finanzierung sorgen, ohne dass die entsprechenden Anstalten hierin eingebunden wären.

\section{Erkenntnisse aus einer Aus- wertung von Fachzeitschriften}

Ergänzt wurde die Befragung der Ministerien und die Durchsicht von Gefangenenzeitungen durch eine Auswertung der Fachzeitschriften nach entsprechenden Beiträgen über Kunstgruppen im Strafvollzug. Auch hier zeigte sich jedoch schnell, dass nur sehr wenig über Kunst im Strafvollzug veröffentlicht wird und die wenigen Veröffentlichungen hierzu ganz überwiegend älteren Datums sind. Eine aktuellere Ausnahme bildet hier die Tagung „Kunsttherapie im Strafvollzug“, die im Juni 2005 in Sachsen veranstaltet wurde ${ }^{7}$. Betrachtet man die Bedeutung, die gerade alternative Formen des Umgangs mit Straffälligen in den letzten Jahren gewinnen, ist dies sehr bedauerlich. Vermutlich liegt dies einerseits an der Struktur der Projekte, die oftmals nur kurze Zeit laufen und nicht langfristig angelegt sind, andererseits möglicherweise auch an mangelndem Interesse seitens der Verantwortlichen.

\section{Projektbeispiele}

Doch auch langfristig laufende Projekte sind nicht immer gut dokumentiert. So findet sich z.B. über Art-i.G. (= Art im Gefängnis), ein seit 1993 unter diesem Namen laufendes Projekt in der JVA für Frauen in Vechta ein Hinweis im internet ${ }^{8}$, in der Fachöffentlichkeit ist die Gruppe jedoch nahezu unbekannt. Art-i.G. organisiert beispielsweise regelmäßig Kunstausstellungen in der JVA Vechta, bei denen sowohl externe als auch interne KünstlerInnen ihre Arbeiten präsentieren können. Hierzu sind zu bestimmten Öffnungszeiten auch interessierte BürgerInnen eingeladen und es geschieht so eine Verknüpfung zwischen Öffentlichkeit und Vollzug, aber auch zwischen Kunst und der real erlebbaren, weil betretbaren Situation der Haftanstalt. In der JVA Fulda besteht seit 15 Jahren durch den Einsatz einer pensionierten Kunstpädagogin eine Malgruppe, die einmal in der Woche zusammen kommt, allerdings nur regional - durch Ausstellungen - bekannt ist. Hier gehen viele Projekte und deren Arbeiten einer größeren Öffentlichkeit leider verloren, bzw. beschränken sich auf Präsentationen und Ausstellungen im regionalen Umfeld.

1. Bildhauerwerkstatt der JVA Bremen. Anders sieht es bei der Bildhauerwerkstatt in der JVA Bremen aus. Diese wurde Ende der 1970er Jahre ins Leben gerufen, nachdem der Künstler Neuenhausen 1976 im Rahmen einer öffentlichen Ausschreibung zu „Kunst im öffentlichen Raum“9 entsprechende Mittel für ein zunächst nur befristet gedachtes Projekt mit Strafgefangenen gewonnen hatte. Sowohl Neuenhausen, als auch später sein Schüler und Nachfolger Dettelbach haben ihre Arbeit in Aufsätzen und Buchveröffentlichungen dokumentiert und so der Öffentlichkeit einen Einblick in die bildhauerische Arbeit mit Strafgefangenen gegeben ${ }^{10}$. Die Bildhauerwerkstatt besteht bis heute; sie ist auch eines der wenigen Beispiele bei denen Gefangene ihrer Arbeitspflicht im Sinne des § 41 StVollzG im Rahmen eines Kunstprojektes nachkommen können und dafür entlohnt werden. Ergänzenswert ist noch, dass die künstlerischen Ergebnisse nicht hinter den Mauern der JVA verbleiben, sondern ihren Weg in den öffentlichen Raum der Bremer Innenstadt (öffentlichen Plätze, Kindergärten, Schulen etc) finden.

2. Projekt Bauarbeiter in der JVA Hannover. Ein kürzeres Projekt Neuenhausens, in den Jahren 1999/2000 in der JVA Hannover angesiedelt, stellte die Gefangenen vor die Aufgabe, lebensgroße Figuren - Bauarbeiter - aus Holz zu schaffen, in unterschiedlicher Kleidung, bei unterschiedlichen Arbeits-Tätigkeiten. Entstanden ist hieraus ein Ensemble von 14 Figuren und einigen Bausstellenutensilien, die auch öffentlich ausgestellt wurden ${ }^{11}$.

3. „Kunst im Knast“ in der JVA Butzbach. Ein weiteres schon seit langem erfolgreich laufendes Projekt ist „Kunst im Knast“ / „Kulturelle Praxis in der JVA Butzbach“12 bzw. seit 1985 unter dem Titel „Kunstpädagogische Praxis im Strafvollzug". Dieses Projekt wurde 1980 auf Anregung eines Gefangenen konzipiert, wobei die Betreuung von Lehrenden und Studierenden des „Instituts für Kunstpädagogik und Visuelle Kommunikation" der Universität in Gießen übernommen wurde. Mitbegründerin war KämpfJansen, die in der Kunstpädagogik das Konzept der Ästhetischen Forschung $^{13}$ als Methode künstlerischen Lernens und Arbeitens entwickelt hat. Inhaltlich ist das Projekt seit seiner Anfangzeit kunstpädagogisch ausgerichtet und es geht bei 
der praktisch-künstlerischen Arbeit der Gefangenen auch um die ästhetische Wahrnehmung, also nicht nur um ein Kunst-machen sondern auch darum, diese kennen zu lernen und $\mathrm{zu}$ verstehen. Vielleicht mehr noch als in anderen Projekten wird hier auch auf das soziale Miteinander Wert gelegt, in der Verbindung zur künstlerischen Arbeit von Gefangenen und BetreuerInnen. Auch hier wie in vielen anderen Projekten haben über die Jahre verschiedene MitarbeiterInnen und -teams die Gruppe begleitet, dabei auch eigene Akzente gesetzt, so dass auch in den Projekten Bewegung ist und diese sich immer wieder wandeln.

4. Kreative Angebote in der JA Neustrelitz. In der Jugendanstalt Neustrelitz (Mecklenburg-Vorpommern) finden sich vielfältige Angebote, so ein Projekt „Bildhauerei im Knast - ein Kurs für Holzgestaltung“, eine externe Kunsttherapiegruppe, ein Theaterworkshop, der von zwei Theaterpädagoginnen durchgeführt wird. Durch Fördergelder einer Stiftung wird hier sogar ein Klavierunterricht angeboten.

\section{Kreative Angebote in der JA} Raßnitz. In der Jugendanstalt Raßnitz (Sachsen-Anhalt) werden von einer katholischen Ordensschwester und der evangelischen Anstaltspfarrerin verschiedene Projekte zum kreativen Arbeiten angeboten, u.a. Zeichnen oder Arbeiten mit Ton und Gips, daneben z.B. aber auch die künstlerische Ausgestaltung der Gefangenenzeitung „Gefangene Gedanken“. Anstaltsübergreifend besteht ein sogenannter „Kreativzirkel“, der sich einmal wöchentlich trifft um neben dem kreativen Arbeiten auch etwas zu lernen. $\mathrm{Zu}$ den Lerninhalten gehören hier z.B. Informationen über Kunstrichtungen und Künstler, aber auch praktische Lernstoffe wie Perspektive, Farbenlehre, Stillehre usw. Die Arbeiten werden innerhalb der Anstalt ausgestellt. Externe Angebote wären, so die Auskunft der JA, ausdrücklich erwünscht, fehlen aber u.a. aufgrund der schlechten verkehrstechnischen Anbindung und einer abgelegenen Lage der Anstalt. In einem Schreiben der JA werden als Kreativ-Gruppen im weiteren Sinn unter anderem genannt: Kirchenchor, Gitarrenkurs, Keyboardunterricht, Dichterlesung und Buchbesprechung, Koch- und Backzirkel, Gruppen zu den Themen Modelleisenbahn, Aquarien- und Terrarien, eine Arbeitsgruppe zur Landschaftsgestaltung und die Betreuung der Anstaltszeitung. Hierin zeigt sich eine fast unüberschaubare Vielfalt an Möglichkeiten, die die Gefangenen nutzen können, um sich vom Haftalltag etwas Abwechslung zu verschaffen.

6. „kunst.voll“ - Kunst im Jugendvollzug der JVA Bremen. Abschließend sei noch auf ein neueres Projekt hingewiesen, das im März 2005 - unabhängig von der oben erwähnten Bildhauerwerkstatt - im Bremer Jugendvollzug als Freizeitgruppe unter dem Namen „kunst.voll“ startete ${ }^{14}$. Das Angebot - zunächst nur Samstags, mittlerweile auch am Sonntag - richtet sich an zu Jugendstrafe verurteilte junge Männer, von denen bis zu neun an der Gruppe teilnehmen können. Zurück geht das Projekt auf eine Idee von Studierenden der Fachhochschule Ottersberg ${ }^{15}$, die sich vorgenommen hatten, mit Strafgefangenen künstlerisch tätig zu sein. „Kunst.voll“ ist - ähnlich dem Butzbacher Projekt - eher pädagogisch denn kunsttherapeutisch ausgerichtet und es geht in der Arbeit auch hier vorrangig um das eigene Erfahren, das gemeinsame Lernen und den richtigen Umgang mit Techniken und Materialien.

\section{Raum für Kreativität in Haft}

Nach diesen ausgewählten Beispielen ist nunmehr genauer zu differenzieren. Es gibt eine Vielzahl von kreativen Projekten, die allerdings zumeist zeitlich befristet sind und die vornehmlich auf Initiative externer Gruppe oder gar einzelner KünstlerInnen zurück gehen. Hierbei handelt es sich dann in der Regel um Freizeitgruppen, d.h. die Angebote finden zumeist nur an einem Tag in der Woche für einige Stunden statt und nicht als Projekt, das den Gefangenen wirklich längerfristig beschäftigen könnte. Im Rahmen von JVA-eigenen Freizeitangeboten bzw. „Angeboten zur Fortbildung in der Freizeit" finden sich ebensolche Möglichkeiten, z.B. Gitarrenunterricht oder Töpferkurse, die dann allerdings nicht die künstlerisches Arbeit primär im Blick haben. Erwähnenswert sind hier im übrigen auch die mittlerweile in zahlreichen Justizvollzugsanstalten angebotenen Schreibwerkstätten, die mal intern, mal von externen Gruppen organisiert werden. Teilweise werden solche Projekte von "Profis“, also ausgebildeten JournalistInnen durchgeführt, es gibt aber auch hier Modelle bei denen Studierende mit den Gefangenen gemeinsam lernen.

Kunstprojekte der Anstalten, bei denen die Anleitenden MitarbeiterInnen der JVA sind, sind vergleichsweise selten. Einzelne Beispiele zeigen jedoch, dass es auch sinnvoll und vor allem möglich sein kann, Gefangene im Rahmen ihrer Arbeitsverpflichtung nach $\S$ 41 StVollzG künstlerisch tätig sein $\mathrm{zu}$ lassen.

\section{Kunsthandwerkliche Betriebe}

In vielen Bundesländern finden sich darüber hinaus Werkstätten, in denen z.B. aus Holz Figuren und Nutzgegenstände hergestellt werden. Hier sind durchaus auch kreative Elemente in der Tätigkeit vorhanden, wenn auch das Ergebnis eher als "Kunsthandwerk" bezeichnet bzw. in Abgrenzung zur „Bildenden Kunst" dann als "Angewandte Kunst" betrachtet werden sollte. Diese Werkstätten verstehen sich allerdings auch nicht als kreative Werkstätten, sondern als Arbeitsbetriebe, die sich der Holzarbeit oder dem Kunsthandwerk widmen ${ }^{16}$. Erstaunlich oft finden sich im Vollzug auch Musikangebote - zwei Bundesländer wiesen ausdrücklich auf Trommelkurse hin, in einem Fall wurde besonders ein Gefangenenchor genannt, der auch öffentliche Konzerte gibt - oder Theatergruppen.

Es gibt auch Länder, in denen künstlerische Projekte von Studierenden durchgeführt werden (ausdrücklich genannt von Hessen, Mecklenburg-Vorpommern und Bremen), sowie solche, bei denen es zu einer Zusammenarbeit mit den Volkshochschulen kommt (BadenWürttemberg, Thüringen).

Ergänzend wurde mehrfach darauf hingewiesen, dass es externe Vereine gibt, die die kreative Arbeit im Vollzug unterstützen, Spenden einwerben und sich um Ausstellungsmöglichkeiten bzw. auch um Katalogveröffentlichungen bemühen. Diese finden sich mit unterschiedlichen Aufgaben und Arbeitsschwerpunkten z.B. Bremen („Mauern öffnen“), Niedersachsen („Cura e.V. Lingen, Verein für Straffälligenhilfe"), Hessen ("Gefangenenhilfe Butzbach“), dem Saarland („Kunst und Kultur im saarländischen Strafvollzug“) und Sachsen („Kunst im Gefängnis“). „Kunst und Knast“ e.V. in Berlin, ein Verein der im Jahr 2005 sein 15jähriges Bestehen feierte, förderte in den Jahren z.B. zahlreiche Mal- und Bildhauerworkshops, Theater- und Musikangebote (von Trommelkursen bis zum Rap), Tanzangebote (allgemeiner Art, aber auch spezieller mit Tango- oder Ausdruckstanz) und initiierte Lesungen von bekannten Schauspielern.

\section{Organisation, Finanzierung und Dokumentation}

Generell zeigt sich, dass fast im Stillen ein doch recht breites Angebot an kreativen Möglichkeiten besteht.

Hierzu zu rechnen sind z.B. auch die zahlreichen Musik- und Theatergruppen. Im Jugendvollzug finden sich auch immer wieder Maskenarbeit, Tanz- und HipHop-Kurse, die den Jugendlichen die Möglichkeit geben sich abzureagieren und bei denen sie gleichzeitig alters- und interessengerecht mit einem Thema angesprochen werden, das sie tatsächlich auch in ihrem Lebensalltag beschäftigt. Hier spielt auch der immer wieder in der Kunsttherapie bemühte Satz hinein, man müsse den Klienten/Patienten dort abholen, wo er sich befindet. Künstlerische und kreative Angebote müssen mithin interessengerecht sein, die Gefangenen ansprechen; dann handelt es sich um Projekte, die mit Nachdruck genutzt werden und auch positive Effekte auf die Teil- 
nehmerInnen, die Gefangenen wie die ProjektbegleiterInnen, haben.

Gemeinsam haben die meisten Angebote, dass es sich um befristete Projekte handelt, deren Anstoß nicht selten von außen kommt und die dann durch den Einsatz von externen MitarbeiterInnen zustande kommen.

Gemeinsam haben diese Projekte allerdings auch, dass die Arbeit in der Regel nicht dokumentier wird und daher über den direkten Umkreis der Gruppe und der JVA hinaus oftmals nichts über die Arbeit bekannt wird. Dies ist umso bedauerlicher, als so auch positive Erfahrungen nicht geteilt werden und viele Gruppen beim Start eines neuen Projektes vor Anforderungen stehen (Organisation, Beschaffung der Mittel, Motivation der Gefangenen) denen sich auch andere Gruppen schon stellen mussten. Hier wäre ein Mehr an Öffentlichkeit, vor allem im wissenschaftlichen Diskurs wünschenswert. Im näheren Umkreis der Projekte wird Öffentlichkeit dadurch geschaffen, dass künstlerische Arbeiten ausgestellt, eingeübte Theaterstücke oder Konzerte vorgeführt werden, wobei dann nicht nur Gefangene, sondern auch interessierte Externe eingeladen sind, daran teilzuhaben.

\section{Therapeutische Bedeutung von Kreativität in Haft}

Ausdrücklich für KunsttherapeutInnen vorgesehene Stellen gibt es im Strafvollzug nach Angaben der meisten Landesministerien nicht. Dies mag einerseits daran liegen, dass die Kunsttherapie als solche noch vergleichsweise jung und damit auch weniger bekannt ist ${ }^{17}$. Anderseits gibt es eine Reihe anderer, zunehmend etablierter Therapieformen, die auch von KunsttherapeutInnen übernommen werden können ${ }^{18}$. Insbesondere zur Ergotherapie und zur Beschäftigungstherapie sind die Grenzen dann oftmals fließend, wenn es nicht um die Rehabilitation nach Verletzungen oder Erkrankungen geht. Um letztgenannte Bereiche wird es im Strafvollzug jedoch nur selten gehen; hier stehen neben der Be- schäftigung, der Tätigkeit selbst, insbesondere die psychische Stärkung und ggf. Behandlung im Blickfeld einer therapeutischen Begleitung. Überschneidungen kann es auch zur Arbeitstherapie geben ${ }^{19}$, wenn handwerkliche Tätigkeiten kunsthandwerkliche Ergebnisse erbringen oder mit künstlerischen Mittel motorische Fähigkeiten einzuüben sind. Im Jugendvollzug können auch Angebote der Verhaltenstherapie und der Erlebnispädagogik durch künstlerische Aufgabenstellungen ergänzt werden. Vielleicht ist mithin der Begriff der „Kunsttherapie“ für Aufgaben des Strafvollzuges zu eng gefasst und es sollte hier mehr auf ein die verschiedenen Therapierichtungen integrierendes Vorgehen gesetzt werden.

Grundsätzlich kann heute jedoch als erwiesen angesehen werden, dass kreative Betätigung positive Auswirkungen auf den Menschen hat ${ }^{20}$. Ganz profan kann dies der Zeitvertreib sein und die Verhinderung von Langeweile. Strafvollzug bietet nur wenige Möglichkeiten für die Gefangenen sich auszuleben und auszudrücken. Malen und Zeichnen - und sei es gerade alleine auf der Zelle - kann hier eine Möglichkeit sein, nicht in Untätigkeit zu versinken. Positive Auswirkungen der künstlerischen Tätigkeit lassen sich auch auf die Psyche des Menschen verzeichnen und helfen, Defizite und Nachteile zu kompensieren. Dies ist umso wichtiger als Strafvollzug erkanntermaßen negative Folgen haben kann. § 3 Abs. 2 StVollzG weist mit dem Gegenwirkungsgrundsatz in der Formulierung „Schädlichen Folgen des Freiheitsentzuges ist entgegenzuwirken" ausdrücklich darauf hin. Diese äußern sich vordergründig auch in psychischen Reaktionen auf die Haft. Zwar ist in Deutschland die Kunsttherapie bislang nur in wenigen Bereichen hinreichend wissenschaftlich belegt und nur für einzelne Krankheitsbilder offiziell medizinisch als Therapie anerkannt. $\mathrm{Zu}$ nennen sind hier insbesondere die Onkologie $\mathrm{e}^{21}$, aber auch Teil-Bereiche der Geriatrie ${ }^{22}$. Jedoch sind auch in vielen anderen Bereichen positive Effekte kreativen Gestaltens schon längst erkannt worden und die Kunsttherapie findet hier seit langem ihren Einsatz. Beispielhaft mag hier die Kunsttherapie bei AIDS-Kranken sein, wie sie z.B. von Niederreiter eindrucksvoll dokumentiert wurde ${ }^{23}$. Hieraus können auch Anregungen folgen, wie sich künstlerische Therapien bei körperlichen Erkrankungen im Strafvollzug einsetzen lassen.

Menschen mit schizophrenen Erkrankungen wie der wohl bis heute bekannteste psychisch kranke Künstler, Adolf Wölfli ${ }^{24}$, haben oftmals umfangreiche Sammlungen von Kunstwerken geschaffen. Erfahrungen gibt es auch mit depressiven Menschen oder mit Menschen, die unter einer bipolaren Störung ( $=$ manische Depression) leiden ${ }^{25}$. Nicht zuletzt finden die Kreativitätstherapien fruchtbaren Anklang in psychiatrischen Kliniken ${ }^{26}$. Hier kann es aber auch Vermischungen mit der Ergo- und Verhaltenstherapie geben und die „reine“ Kunsttherapie ist weit seltener zu finden. Auch müssen psychische Störungen nicht den Grad des Krankheitswertigen erreichen, um sie mit künstlerischen Mitteln zu bearbeiten. Besser ist es, Probleme schon im Vorfeld aufzufangen, bevor diese sich manifestieren können. Es gibt mithin gute Argumente, Strafgefangenen entsprechende Angebote zu machen, um vielleicht schon frühzeitig den negativen psychischen Folgen der Haft einen positiv wirkenden Rückhalt entgegenzusetzen.

\section{Besitz und Beschaffung künstlerischen Materials}

Das deutsche Strafvollzugsgesetz (und die kommenden Landesgesetze, soweit derzeit ersichtlich) kennt den Begriff der Kunst nicht und sieht mithin auch nicht vor, dass ein Gefangener einen Rechtsanspruch darauf hätte, sich künstlerisch zu betätigen. Anders sieht dies beispielsweise im Österreichischen Strafvollzugsgesetz (StVG) aus. Hier sieht § 63 StVG ausdrücklich sogar einen Rechtsanspruch auf Besitz von Gegenständen vor, die der kreativen Betätigung dienen. Wörtlich heißt es „Die Strafgefangenen sind berechtigt, in der Freizeit in angemessenem Umfang zu zeichnen, zu malen oder sich sonst bildnerisch zu betätigen." Nach h.M. begründet sich hieraus ein subjektiv-öffentliches Recht des Strafgefangenen ${ }^{27}$, das z.B. auch das Schnitzen oder das Herstellen von Drucken umfasst. Eingeschränkt werden kann dieses Recht nur in engen Grenzen, nämlich dann wenn Missbrauch der Materialien oder des Produktes (z.B. nationalsozialistische Symbole) zu befürchten ist ${ }^{28}$.

In Deutschland gibt es einen solchen Rechtsanspruch nicht, der Gefangene muss mithin - auch auf die Gefahr einer Ablehnung hin - die jeweiligen Gegenstände ausdrücklich beantragen. In der Regel wird ihm dies nicht versagt werden, und vielfach sehen schon die Hausordnungen vor, dass der Gefangene Papier und Stifte besitzen darf. Eine Klarstellung eines solchen Anspruches auch in Deutschland könnte entsprechende Verfahren jedoch vereinfachen. Die im Rahmen des vorliegenden Projektes gewonnenen Rückmeldungen zeigen dabei auch sehr deutlich, dass kreatives Arbeiten sowohl seitens der Aufsichtsbehörden als auch der Anstalten nach Kräften unterstützt wird. Offene Diskussionspunkte gibt es allenfalls 1. bei der Frage, ob künstlerisches Tätigsein als Pflichtarbeit im Sinne des $\S 41$ StVollzG anerkannt und entlohnt wird und 2. wann und unter welchen Voraussetzungen es einem künstlerisch besonders begabtem Gefangenen gestattet werden kann, im Rahmen des § 39 Abs. 2 StVollzG selbstbeschäftigt zu arbeiten. Grundsätzliche Ablehnungen des Besitzes von künstlerischem Material gibt es hingegen nicht, im Einzelfall kann dies - z.B. bei Aufgaben der Bildhauerei - aber an organisatorische Grenzen stoßen.

\section{Resümee und Ausblick}

Kreatives Tätigsein bietet auch im Strafvollzug ein enormes, teilweise noch sehr unterschätztes Potential. Insofern kann auch hier die Neuenhausen'sche Frage nach „Luxus oder Notwendigkeit" eindeutig beantwortet werden. Das Vermögen sich kreativ ausdrücken zu können muss auch im Strafvollzug unabdingbar zum Leben dazugehören und ist insofern notwendig und alles andere 
als überflüssiger Luxus. Eine Möglichkeit ist die Selbstbeschäftigung mit Stift, Farbe und Papier. Wichtiger aber noch ist es, die kreativen und künstlerischen Möglichkeiten der Gefangenen durch entsprechende Angebote weiter zu fördern.

Tatsächlich sieht es um die Kunst in Haft auch nicht so düster aus, wie ein erster Blick in die Literatur und kursorische Umfragen es erscheinen lassen.

Viele Projekte finden eher im "Verborgenen“ der Anstalten statt, entfalten dort ihren Nutzen für die Gefangenen und nicht zuletzt auch für die KünstlerInnen, die sich hierauf einlassen. Zumeist werden sie aber nicht nach außen getragen oder erreichen durch Vorführungen und Ausstellungen allenfalls regionale Bekanntheit. Dies verfälscht das Bild und lässt auch andere Menschen nicht an den so gewonnenen Erfahrungen teilhaben.

Kunst und Kreativität haben ihren Raum auch im Strafvollzug. Dabei mag als zweiter Aspekt hinzukommen, dass nicht alles was Kunst und Kreativität genannt werden könnte auch tatsächlich so genannt wird. Manch ein kreatives Angebot wie eine Musikgruppe oder ein Töpferkurs wird gar nicht als Kunst verstanden, wie auch Angebote der Ergotherapie oder der Beschäftigungstherapie vielleicht sehr viel mehr kreative Aspekte beinhalten, als aus dem therapeutischen Schubladendenken deutlich wird. Auch aus der Umfrage bei den Landesministerien wurde deutlich, wie vielfältig die Angebote und Möglichkeiten sind, wenn von handwerklichen über musikalische bis hin zu Theatergruppen Beispiele für kreative Arbeit in Haft genannt wurden.

So steht ein Überblick über künstlerische und kreative Angebote im Strafvollzug auch weiter aus und ist vielleicht ob der Vielzahl der Angebote, aber auch ob der Kürze der meisten Projekte kaum leistbar.

Wünschenswert ist in jedem Fall mehr über entsprechende Arbeiten $\mathrm{zu}$ lesen, um sich so fachlich besser austauschen aber auch etwas über die Potentiale kreativen Tuns in Haft lernen zu können.

\section{Literatur:}

Ammann, Jean Ch. u.a., Kunst im Knast 2. Projekt Kunst im Strafvollzug in der Justizvollzugsanstalt Butzbach seit 1981, Eigenverlag der Gefangenenhilfe Butzbach e.V. 2006

Bammann, Kai, Kein Schritt vorwärts, drei zurück? Aktuelle Entwicklungen im Strafvollzug(-srecht): Angriff auf Vollzugsziel, Lockerungen und die Bundeskompetenz, in: forum recht Heft 3 , 2006, S. 81-83

Bammann, Kai, Kunst im Jugendstrafvollzug. Ansätze aus der Arbeit der Projektgruppe „kunst.voll“ in der JVA Bremen, in: ZJJ Heft 2/2007, S. 206 ff.

Biermann, Marietta, Eurythmie im Knast. Bericht aus Bonn, in: Flensburger Hefte, Heft 27, 1989, S. 75-82

Dettelbach, Bernd-Wolf, Kolonne Kunst. Ein Bericht über 1528 Stunden Bildhauerei mit Gefangenen, Braunschweig: Hinz \& Kunst 1986

Drexler, Karl, Strafvollzugsgesetz (StVG), Wien: Manz'sche Verlagsbuchhandlung, 2003

Ehmer, Hermann K./ Kämpf-Jansen, Helga (Hrsg.), Kunst im Knast 1980-1985. Dokumente ästhetischer und sozialer Erfahrung, Giessen, Eigenverlag der Gefangeneninitiative Butzbach e.V. 1985

Fuchs, Thomas, Depression und Manie, in: Spreti, Flora von u.a. (Hrsg.) Kunsttherapie bei psychischen Störungen, München und Jena: Urban \& Fischer 2005, S. 81-87

Gan $\beta$, Michael/Linde, Matthias (Hrsg.), Kunsttherapie mit demenzkranken Menschen, Mabuse-Verlag, Frankfurt 2004

Haberkorn, Alfred u.a. (Bearb.), Kunsttherapie im Strafvollzug.Tagung vom 6.-8. Juni 2005 in Bautzen, Tagungsdokumentation, Eigenverlag der JVA Waldheim 2006

Henn, Wolfgang/Gruber, Harald (Hrsg.), Kunsttherapie in der Onkologie, ClausRichter-Verlag, Köln 2004

Herlitz, Michael u.a., Kunst im Knast. Projekt Kunst im Strafvollzug in der Justizvollzugsanstalt Butzbach seit 1981, Eigenverlag der Gefangenenhilfe Butzbach e.V. 2001

Kämpf-Jansen, Helga, Ästhetische Forschung. Wege durch Alltag, Kunst und Wissenschaft. $\mathrm{Zu}$ einem innovativen Konzept ästhetischer Bildung, Salon Verlag, Köln 2000

Manske, Hans-Joachim, Kunst im öffentlichen Raum in Bremen. Die Entwicklung eines Programms, Dokumentation 1977-1980, Schmalfeldt-Verlag, Bremen 1980

McNiff, Shaun, Art as medicine. Creating a therapy of the imagination, Boston and London, Shambhala 1992

Mecher-Schönach, Christine, Kunsttherapie und ihre 47 Namen..., in: Spreti, Flora von u.a. (Hrsg.) Kunsttherapie bei psychischen Störungen, München und Jena: Urban \& Fischer 2005, S. 10-12
Morgenthaler, Walter, Ein Geisteskranker als Künstler. Adolf Wölfli, Medusa-Verlag, Wien und Berlin, Neuausgabe 1985 (Original: 1921)

Neuenhausen, Siegfried u.a., Die Arbeit von Künstlern mit Gefangenen in Justizvollzugsanstalten, Ms. im Auftrag des Bundesministeriums der Justiz, Bonn 1981

Neuenhausen, Siegfried, Graben nach verschütteter Kreativität. Siegfried Neuenhausens Kunstprojekte mit Gefangenen in Bremen, Patienten in Wunstorf und Ochsenzoll, Behinderten in Braunschweig, Braunschweig: Eigenverlag der Hochschule für Bildende Künste 1992

Neuenhausen, Siegfried, Bauarbeiter ein Figurenensemble. Bildhauerprojekt mit Gefangenen, Hannover: Eigenverlag 2000

Niederreiter, Lisa, Bilder zwischen Leben, Krankheit und Tod. Künstlerisches Arbeiten und Therapie mit einem an AIDS Erkrankten, Claus-Richter-Verlag Köln 1995

Pütz, Rose-Maria/Pütz, Siegfried, Kunsttherapeut/ Kunsttherapeutin. Blätter zur Berufskunde Band 2, 1. Aufl. Nürnberg 1977 (5. Auflage 1989)

Spoerri, Elka (Hrsg.), Der Engel des Herrn im Küchenschurz. Über Adolf Wölfli, Frankfurt a.M. Fischer 1987

Spreti, Flora von, Kunsttherapie mit depressiven Patienten, in: Spreti, Flora von u.a. (Hrsg.) Kunsttherapie be psychischen Störungen, München und Jena: Urban \& Fischer 2005a, S. 88-103

Spreti, Flora von, Kunsttherapie mit manischen Patienten, in: Spreti, Flora von u.a. (Hrsg.) Kunsttherapie bei psychischen Störungen, München und Jena: Urban \& Fischer 2005b, S 107-117

Tretter, Felix/Bender, Wolfram (Hrsg.), Kunsttherapie in der Psychiatrie, Köln Claus-Richter Verlag 1995

Wattenberg, Heinz-H., Kunst im Strafvollzug. 16 Jahre Erfahrung in der Arbeitsund Beschäftigungstherapie, in: ZfStrVo 1994, S. 288-290

Dr. Kai Bammann, Dipl.-Kriminologe, ist wissenschaftlicher Mitarbeiter am Fachbereich Rechtswissenschaft an der Universität Bremen.

Dr. Johannes Feest, Prof. im Ruhestand, ist Leiter des Strafvollzugsarchivs an der Universität Bremen.

\section{Fußnoten}

1 Neuenhausen u.a. 1981

2 s. Bammann 2006

3 www.strafvollzugsarchiv.de

4 Eurythmie ist eine Verbindung aus Tanz und Bewegung, die aus der Anthroposophie Rudolf Steiners kommt und von ihm ausdrücklich seinerzeit als „neue Bewegungs-Kunst“ verstanden wurde. Zur Eurythmiegruppe in der JVA Bonn siehe Biermann 1989.

4 Dieses Bild sähe im Übrigen für Einrichtungen des Maßregelvollzuges und der (forensischen) Psychiatrie die den Gesundheitsressorts unterstellt sind und die hier nicht nachgefragt wurden, vermutlich deutlich anders aus.
5 http://babe.informatik.uni-bremen de/mediawiki/index.php/Projekt zeitung_\%E2\%80\%9ELohnstufe 4\%E2\%80\%9C/Lohnstufe_4/5_M $\%$ C3\%A4rz 2007

6 Dazu ist zwischenzeitlich eine Dokumentation erschienen, Haberkorn u.a. 2006

7 Art-i.G. (http://www.oldenburgischelandschaft.de/print.php?pv=41\& did=412)

8 nachgewiesen bei Manke 1980

9 Dettelbach 1986, ebenso die Beiträge verschiedener Autoren in Neuenhausen 1992, S. 12-116

10 Neuenhausen 2002

11 Ehmer/Kämpf-Jansen 1985, Herlitz u.a. 2001, Ammann u.a. 2006

12 Kämpf-Jansen 2000

13 siehe für Details Bammann 2007

14 www.fh-ottersberg.de bzw. www. kunsttherapieforschung.de für wissenschaftliche Forschung im Bereich der (anthroposophischen) Kunsttherapie

$15 \mathrm{vgl}$. hierzu beispielhaft das Angebot des Niedersächsischen JVA-Shops im internet unter: http://www.jvaonline-shop.de/ ; hier können im Übrigen über den Verein Cura e.V. auch Bilder erstanden werden, die in niedersächsischen JVAen von männlichen und weiblichen Gefangenen gemalt wurden.

16 Neuenhausen u.a. 1981 erwähnen in ihrer Studie die therapeutischen Effekte des künstlerischen Arbeitens auch nur am Rande und nur im $\mathrm{Zu}$ sammenhang mit der Drogentherapie, ebd. S. 239 f., was nicht überraschend ist da die „Entdeckung“ der Kunsttherapie in Deutschland erst einige Jahre später richtig eingesetzt hat. Zum ersten Mal Eingang in den offiziellen deutschen Sprachgebrauch hat die Berufsbezeichnung „Kunsttherapeut / Kunsttherapeutin" vermutlich 1977 mit Pütz/Pütz 1977 in den von der Bundesanstalt für Arbeit herausgegebenen Blättern zur Berufskunde gefunden.

17 Interessant ist in diesem Zusammenhang vor allem auch der Text von Mechler-Schönach 2005, die recherchiert hat, dass in Deutschland allein 47 verschiedene Namen Verwendung fanden und finden (einschließlich diverser Bindestrich-Kunsttherapien), wobei allerdings anzumerken ist, dass es auch tatsächliche inhaltliche Unterschiede gibt.

$18 \mathrm{Vgl}$. Wattenberg 1994, S. $288 \mathrm{ff}$

19Statt vieler: McNiff 1992

20 Henn/Gruber 2004

$21 \mathrm{Ganß/Linde} 2004$

22 Niederreiter 1995

23 hierzu besonders die grundlegende Studie von Morgenthaler 1985, erstmals erschienen 1921; für weitere Informationen und Nachweise siehe Spoerri 1987

24 Fuchs 2005, S. 81 ff., von Spreti 2005a und dies. 2005b

25 Tretter/ Bender 1995

26 Drexler 2003, § 63 Rz. 1

27 Drexler 2003, ebd. 